\title{
Clustered Star Formation in the Magellanic Clouds
}

\author{
Dimitrios A. Gouliermis \\ Max-Planck-Institute for Astronomy, Königstuhl 17, 69117 Heidelberg, Germany \\ email: dgoulier@mpia.de
}

\begin{abstract}
The Large and Small Magellanic Cloud (LMC, SMC) offer an outstanding variety of young stellar associations, in which large samples of low-mass stars (with $M \leqslant 1 \mathrm{M}_{\odot}$ ) currently in the act of formation can be resolved and explored sufficiently with the Hubble Space Telescope. Previous observations with the Wide-Field Planetary Camera 2 (WFPC2) provided the first evidence of the existence of low-mass pre-main sequence (PMS) stars in the vicinity of star forming associations in the Magellanic Clouds (MCs) (Gouliermis et al. 2006a), and recent results from deeper observations with the Advanced Camera for Surveys (ACS) enhanced dramatically the picture of these systems with the discovery of large numbers of PMS stars. The associations LH 95 (Gouliermis et al. 2002, 2007a) in the LMC, and NGC 346 (Gouliermis et al. 2006b) and NGC 602 (Gouliermis et al. 2007b) in the SMC, are currently under investigation with the use of observations from both Hubble and Spitzer Space Telescope. I present the impact of our recent results in terms of the star formation history and Initial Mass Function (IMF) of these interesting systems, using as example the case of NGC 602.
\end{abstract}

Keywords. Magellanic Clouds - Hertzsprung-Russell diagram — stars: low-mass — stars: pre-main-sequence - stars: formation — stars: evolution - HII regions — galaxies: star clusters — open clusters and associations: individual (LH 95, NGC 346, NGC 602)

\section{Pre-Main-Sequence Stars in Associations of the Magellanic Clouds}

The loci of the low-mass PMS stars in the color-magnitude diagrams (CMD) of these associations show a broadening, which implies continuous star formation for up to $\sim 10 \mathrm{Myr}$. However, there are important factors, such as differential reddening, binarity and variability, that affect significantly the positions of these stars in the CMD, giving false evidence of an age-spread (Hennekemper et al. 2007). Still, our simulations showed that a second or third generation of star formation cannot be excluded for these PMS stars (Hennekemper et al. 2007; Schmalzl et al. 2007). Specifically, in the case of NGC 602 (Fig. 1), if a mean extinction, as it is measured by the displacement of the upper MS stars in comparison to the ZAMS is assumed, then the observed PMS widening can be successfully reproduced in a synthetic CMD if at least two epochs of star formation are considered. On the other hand, if the extinction is higher (which is the case for highly embedded clusters), then a single star formation event would be sufficient to explain the star formation history of this system. However, in this case the system should have an age of the order of $\sim 10 \mathrm{Myr}$, much older than what has been previously claimed ( $\sim 4 \mathrm{Myr})$.

Naturally, the uncertainty in the age of the PMS stars has significant implications to the construction of the IMF of the low-mass stars in the system. Consequently, although for the massive part of the IMF a mass-luminosity relation was considered based on young MS isochrones, the low-mass part was constructed by counting the PMS stars between evolutionary tracks, since there is no specific indication about their age. The constructed IMF is shown in Fig. 1 (left). Further details are available from Schmalzl et al. (2007). 

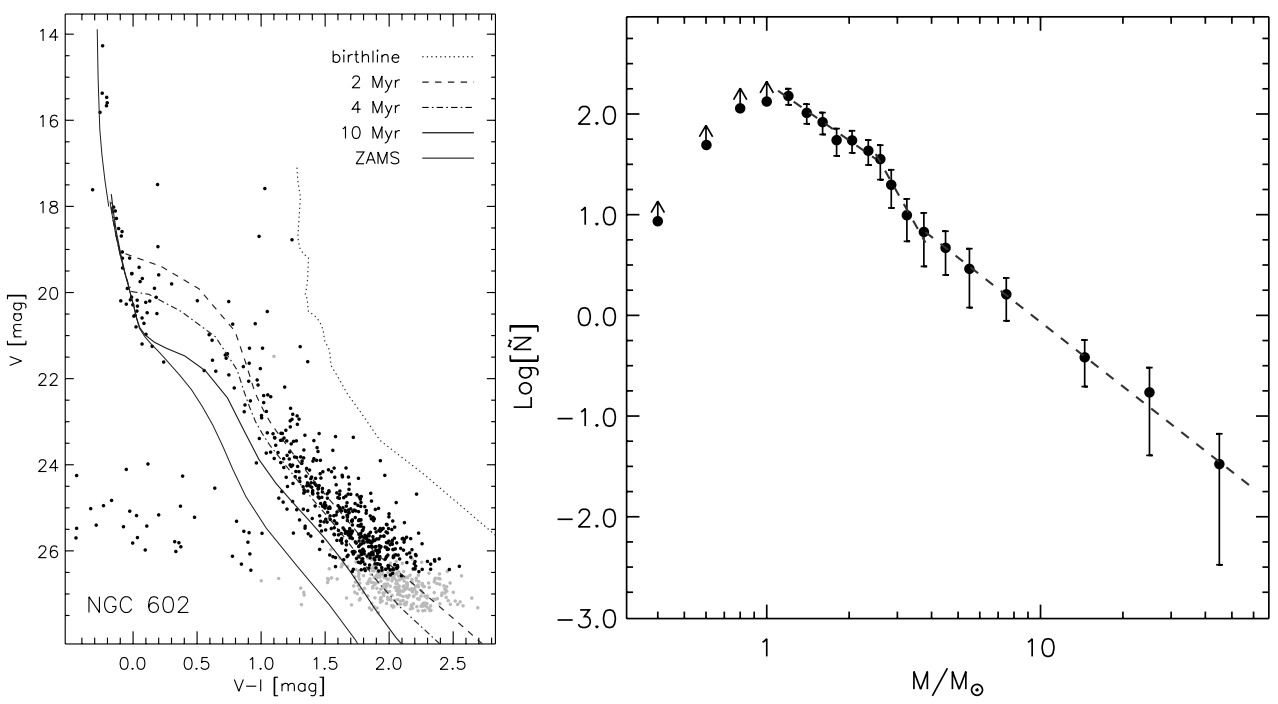

Figure 1. Left: The $V-I, V$ CMD of NGC 602 constructed from photometry with ACS, after the contribution of the general SMC field has been statistically subtracted. It is shown that the stellar association consists of bright MS stars and a large number of low-mass PMS stars. The observed wide spread of the positions of the latter in the CMD, cannot be explained by differential reddening or binarity alone. An alternative explanation is that, assuming that the extinction is relatively low, multi-epoch star formation has taken place, so that the observed widening of the PMS stars is evidence of an age-spread in NGC 602. Three PMS isochrones are overplotted as the most representative of such sequential star formation. Right: The Initial Mass Spectrum of NGC 602 constructed with the use of different methods for the massive MS and the low-mass PMS stars. We find that a slope $\Gamma \simeq-1.4 \pm 0.2$ represents well the IMF of NGC 602. Both plots from Schmalzl et al. (2007).

\section{Acknowledgements}

The author kindly acknowledges the support of the German Research Foundation (DFG) through the individual grant GO 1659/1-1. Based on observations made with the NASA/ESA Hubble Space Telescope, obtained from the data archive at the Space Telescope Science Institute, which is operated by the Association of Universities for Research in Astronomy, Inc. under NASA contract NAS 5-26555. Research supported by the German Research Foundation (DFG), and the German Aerospace Center (DLR).

\section{References}

Gouliermis, D., Keller, S. C., de Boer, K. S., Kontizas, M., \& Kontizas, E. 2002, A\&্A 381, 862

Gouliermis, D., Brandner, W., \& Henning, T. 2006a, ApJ (Letters) 636, L133

Gouliermis, D. A., Dolphin, A. E., Brandner, W., \& Henning, T. 2006b, ApJS 166, 549

Gouliermis, D. A., et al. 2007a, ApJ (Letters) 665, L27

Gouliermis, D. A., Quanz, S. P., \& Henning, T. 2007b, ApJ 665, 306

Hennekemper, E., et al. 2007, Accepted for publication in ApJ (arXiv:0710.0774)

Schmalzl, M., et al. 2007, Submitted to ApJ 